\title{
On Lie ideals and generalized derivations of *-prime rings
}

\author{
Shuliang Huang and Öznur Gölbaşi
}




\title{
ON LIE IDEALS AND GENERALIZED DERIVATIONS OF *-PRIME RINGS
}

\author{
SHULIANG HUANG AND ÖZNUR GÖLBAŞI \\ Received 4 February, 2013
}

\begin{abstract}
Let $(R, *)$ be a 2-torsion free $*$-prime ring with involution $*$ and center $Z(R), U$ a nonzero square closed $*$-Lie ideal of $R$. An additive mapping $F: R \rightarrow R$ is called a generalized derivation if there exits a derivation $d: R \rightarrow R$ such that $F(x y)=F(x) y+x d(y)$. In the present paper, we prove that $U \subseteq Z(R)$ if any one of following conditions holds: 1$)[F(u), u]=0$, 2) $[d(u), F(v)]=0,3) d(u) o F(v)=0,4)[d(u), F(v)]= \pm[u, v], 5) d(u) o F(v)= \pm u o v, 6)$ $d(u) F(v) \pm u v \in Z(R)$, for all $u, v \in U$. Furthermore, an example is given to demonstrate that the $*$-primeness hypothesis is not superfluous.
\end{abstract}

2010 Mathematics Subject Classification: 16W25; 16W10; 16N60; $16 \mathrm{U} 80$

Keywords: $*$-prime rings, Lie ideals, derivations, generalized derivations

\section{INTRODUCTION}

Let $R$ will be an associative ring with center $Z$. For any $x, y \in R$ the symbol $[x, y]$ represents commutator $x y-y x$. Recall that a ring $R$ is prime if $x R y=0$ implies $x=0$ or $y=0$. An additive mapping $*: R \rightarrow R$ is called an involution if $(x y)^{*}=y^{*} x^{*}$ and $\left(x^{*}\right)^{*}=x$ for all $x, y \in R$. A ring equipped with an involution is called a ring with involution or $*$-ring. A ring with an involution is said to $*$-prime if $x R y=x R y^{*}=0$ or $x R y=x^{*} R y=0$ implies that $x=0$ or $y=0$. Every prime ring with an involution is $*$-prime but the converse need not hold general. An example due to Oukhtite [7] justifies the above statement. That is, let $R$ be a prime ring, $S=R \times R^{o}$ where $R^{o}$ is the opposite ring of $R$. Define involution $*$ on $S$ as $*(x, y)=(y, x) . S$ is $*$-prime, but not prime. This example shows that every prime ring can be injected in a $*$-prime ring and from this point of view $*-$ prime rings constitute a more general class of prime rings. In all that follows the symbol $S_{a_{*}}(R)$, first introduced by Oukhtite, will denote the set of symmetric and skew symmetric elements of $R$, i.e. $S_{a_{*}}(R)=\left\{x \in R \mid x^{*}= \pm x\right\}$.

An additive subgroup $U$ of $R$ is said to be a Lie ideal of $R$ if $[U, R] \subseteq U$. A Lie ideal is said to be a $*$-Lie ideal if $U^{*}=U$. If $U$ is a Lie (resp. $*$-Lie) ideal of $R$, then $U$ is called a square closed Lie (resp. $*$-Lie) ideal of $R$ if $x^{2} \in U$ for all $x \in U$. An 
additive mapping $d: R \rightarrow R$ is called a derivation if $d(x y)=d(x) y+x d(y)$ holds for all $x, y \in R$. For a fixed $a \in R$, the mapping $I_{a}: R \rightarrow R$ given by $I_{a}(x)=[a, x]$ is a derivation which is said to be an inner derivation. Recently, in [4], Bresar defined the following notation. An additive mapping $F: R \rightarrow R$ is called a generalized derivation if there exists a derivation $d: R \rightarrow R$ such that

$$
F(x y)=F(x) y+x d(y), \text { for all } x, y \in R .
$$

Basic examples are derivations and generalized inner derivations (i.e., maps of type $x \rightarrow a x+x b$ for some $a, b \in R$ ). One may observe that the concept of generalized derivations includes the concept of derivations and of the left multipliers (i.e., $F(x y)=F(x) y$ for all $x, y \in R)$. Several authors studied commutativity in prime and semiprime rings admitting derivations and generalized derivations which satisfy appropriate algebraic conditions on suitable subsets of the rings. Generalized derivations have been primarily studied on operator algebras. Therefore any investigation from algebraic point of view might be interesting. Recently, some well-known results concerning prime rings have been proved for $*$-prime ring by Oukhtite et al. (see, [5-9], where further references can be found). In [1] the authors explored the commutativity of the ring $R$ satisfying one of the following conditions: (i) $d(x) o F(y)=0$, (ii) $[d(x), F(y)]=0$, (iii) $d(x) o F(y)=x o y$, (iv) $d(x) o F(y)=$ $-x o y$, (v) $d(x) F(y)-x y \in Z$, (vi) $d(x) F(y)+x y \in Z$, (vii) $[d(x), F(y)]=[x, y]$ and (viii) $[d(x), F(y)]=-[x, y]$, for all $x, y$ in some appropriate subset of the ring $R$. Motivated by this fact, we extend these theorems to a nonzero square closed $*-$ Lie ideal $U$ and a generalized derivation of $*$-prime rings.

\section{Preliminaries}

Throughout the paper, we make some extensive use of the basic commutator identities:

$$
\begin{aligned}
& {[x, y z]=y[x, z]+[x, y] z} \\
& {[x y, z]=[x, z] y+x[y, z]} \\
& x o(y z)=(x o y) z-y[x, z]=y(x o z)+[x, y] z \\
& (x y) o z=x(y o z)-[x, z] y=(x o z) y+x[y, z] .
\end{aligned}
$$

Moreover, we shall require the following lemmas.

Lemma 1 (Lemma 4 in [8]). Let $R$ be $a *$-prime ring with characteristic not two, $U$ be a nonzero $*$-Lie ideal of $R$ and $a, b \in R$. If $a U b=a^{*} U b=0$, then $a=0$ or $b=0$ or $U \subseteq Z$.

Lemma 2 (Lemma 2.7 in [2]). Let $R$ be a 2-torsion free $*$-prime ring and $U$ be $a *-$ Lie ideal of $R$. If $a \in R$ such that $[a, U] \subseteq Z$, then either $U \subseteq Z$ or $a \in Z$.

The following Lemma is immediate consequence of Lemma 2.

Lemma 3. Let $R$ be a $*$-prime ring with characteristic not two, $U$ be a nonzero $*-$ Lie ideal of $R$. If $[U, U] \subseteq Z$, then $U \subseteq Z$. 
Lemma 4 (Lemma 2.4 in [6]). Let $R$ be a 2-torsion free $*$-prime ring and $U$ a nonzero $*-$ Lie ideal of $R$. If $R$ admits nonzero derivation $d$ which commutes with $*$ such that $d(U) \subset Z$, then $U \subseteq Z$.

Lemma 5 (Theorem 1.1 in [6]). Let $R$ be a 2-torsion free $*$-prime ring, $U$ a nonzero $*-$ Lie ideal of $R$. If $R$ admits nonzero derivation $d$ which commutes with $*$ such that $d^{2}(U)=0$, then $U \subseteq Z$.

\section{RESULTS}

The following theorem gives a generalization of Posner's well known result [10, Theorem 1].

Theorem 1. Let $R$ be a 2-torsion free *-prime ring, $U$ a nonzero square closed $*-$ Lie ideal of $R$. If $R$ admits derivations $d_{1}, d_{2}$ such that $* d_{2}=d_{2} *$ and $d_{1} d_{2}(U)=$ 0 , then either $d_{1}=0$ or $d_{2}=0$ or $U \subseteq Z$.

Proof. Notice that $u v+v u=(u+v)^{2}-u^{2}-v^{2}$, for all $u, v \in U$. Since $u^{2} \in U$ for all $u \in U, u v+v u \in U$. Also $u v-v u \in U$, for all $u, v \in U$. Hence, we get $2 u v \in U$, for all $u, v \in U$.

Now, we have $d_{1} d_{2}(u)=0$, for all $u \in U$. Replacing $u$ by $2 u v$ and using $R$ is a 2-torsion free, we have $d_{1}\left(d_{2}(u) v+u d_{2}(v)\right)=0$, and hence

$$
d_{2}(u) d_{1}(v)+d_{1}(u) d_{2}(v)=0 \text {, for all } u, v \in U .
$$

Again replace $v$ by $v d_{2}(w), w \in[U, U]$ to get

$$
d_{1}(u) v d_{2}^{2}(w)=0, \text { for all } u, v \in U, w \in[U, U] .
$$

Since $U$ is a nonzero square closed $*$-Lie ideal of $R$, we have $[U, U]$ is a nonzero square closed $*$-Lie ideal of $R$, too. Hence using $* d_{2}=d_{2} *$ the last equation, we arrive at

$$
d_{1}(u) U d_{2}^{2}(w)=d_{1}(u) U *\left(d_{2}^{2}(w)\right)=0, \text { for all } u \in U, w \in[U, U] \cap S_{a_{*}}(R)
$$

By Lemma 1 , we get either $d_{1}(u)=0$, for all $u \in U$ or $d_{2}^{2}(w)=0$ for each $w \in$ $[U, U] \cap S_{a_{*}}(R)$.

If $d_{1}(u)=0$, for all $u \in U$, then we have $d_{1}=0$ or $U \subseteq Z$ by Lemma 4. Assume that $d_{2}^{2}(w)=0$ for each $w \in[U, U] \cap S_{a_{*}}(R)$. Let $w \in[\bar{U}, U]$ as $w+w^{*}, w-w^{*} \in$ $[U, U] \cap S_{a_{*}}(R)$ and $d_{2}^{2}(w)=0$. Hence we have $d_{2}^{2}([U, U])=0$, then we get $d_{2}=$ 0 or $[U, U] \subseteq Z$ by Lemma 5 , and so $U \subseteq Z$ by Lemma 3 . This completes the proof.

Theorem 2. Let $R$ be a 2-torsion free $*$-prime ring, $U$ a nonzero square closed $*-$ Lie ideal of $R$. If $R$ admits a nonzero generalized derivation $F$ associated with nonzero derivation $d$ which commutes with $*$ such that $[F(u), u]=0$, for all $u \in U$ then $U \subseteq Z$. 
Proof. Suppose that

$$
[F(u), u]=0, \text { for all } u \in U .
$$

Linearizing (3.1) and using this, we obtain

$$
[F(u), v]+[F(v), u]=0, \text { for all } u, v \in U .
$$

Replacing $v$ by $2 v u$ in (3.2) and using $R$ is a 2-torsion free, we get

$$
[F(u), v u]+[F(v) u+v d(u), u]=0 .
$$

That is

$$
\begin{aligned}
& {[F(u), v] u+v[F(u), u]+[F(v), u] u+} \\
& F(v)[u, u]+v[d(u), u]+[v, u] d(u) \\
& =0, \text { for all } u, v \in U .
\end{aligned}
$$

Now combining (3.1) and (3.2), using hypothesis in the last equation, we find that

$$
v[d(u), u]+[v, u] d(u)=0, \text { for all } u, v \in U .
$$

Again replace $v$ by $2 v w$ in (3.3) and use (3.3), to get

$$
[v, u] w d(u)=0, \text { for all } u, v, w \in U .
$$

and so

$$
[v, u] U d(u)=0 \text {, for all } u, v \in U .
$$

Since $U$ is a nonzero $*$-Lie ideal of $R$ yields that

$$
[v, u]^{*} U d(u)=0 \text {, for all } v \in U, u \in U \cap S_{a_{*}}(R) .
$$

By Lemma 1, we get either $[v, u]=0$, for all $v \in U$ or $d(u)=0$ for each $u \in$ $U \cap S_{a_{*}}(R)$. Let $u \in U$, as $u+u^{*}, u-u^{*} \in U \cap S_{a_{*}}(R)$ and $\left[v, u \pm u^{*}\right]=0$, for all $v \in U$ or $d\left(u \pm u^{*}\right)=0$. Hence we have $[v, u]=0$ or $d(u)=0$, for all $u, v \in U$. We obtain that $U$ is union of two additive subgroups of $U$ such that

$$
K=\{u \in U \mid d(u)=0\}
$$

and

$$
L=\{u \in U \mid[v, u]=0, \text { for all } v \in U\} .
$$

Morever, $U$ is the set-theoretic union of $K$ and $L$. But a group can not be the settheoretic union of two proper subgroups. Hence $K=U$ or $L=U$. In the former case, we get $U \subseteq Z$ by Lemma 4. In the latter case, $[U, U]=(0)$. That is $U \subseteq Z$ by Lemma 3. This completes the proof.

Corollary 1. Let $R$ be a 2 -torsion free $*$-prime ring, $U$ a nonzero square closed $*-$ Lie ideal of $R$. If $R$ admits a nonzero derivation $d$ which commutes with $*$ such that $[d(u), u]=0$, for all $u \in U$ then $U \subseteq Z$. 
Theorem 3. Let $R$ be a 2-torsion free $*$-prime ring and $U$ a nonzero square closed $*-$ Lie ideal of $R$. If $R$ admits a nonzero generalized derivation $F$ associated with nonzero derivation $d$ which commutes with $*$ such that $[d(u), F(v)]=0$, for all $u, v \in U$, then $U \subseteq Z$.

Proof. We have

$$
[d(u), F(v)]=0, \text { for all } u, v \in U .
$$

Replacing $u$ by $2 u w, w \in U$ in (3.5) and applying (3.5), we get

$$
2(d(u)[w, F(v)]+[u, F(v)] d(w))=0, \text { for all } u, v, w \in U .
$$

Using the fact that $R$ is a $2-$ torsion free ring, we have

$$
d(u)[w, F(v)]+[u, F(v)] d(w)=0, \text { for all } u, v, w \in U .
$$

Substituting $d(t), t \in[U, U]$ for $w$ in (3.6), we get

$$
d(u)[d(t), F(v)]+[u, F(v)] d^{2}(t)=0, \text { for all } u, v \in U, t \in[U, U] .
$$

Using the hypothesis, the above relation yields that

$$
[u, F(v)] d^{2}(t)=0, \text { for all } u, v \in U, t \in[U, U] .
$$

Now, taking $2 v u$ instead of $u$ in (3.7) and using this equation, $\operatorname{char} R \neq 2$, there by obtain

$$
[v, F(v)] U d^{2}(t)=0, \text { for all } v \in U, t \in[U, U] .
$$

Since $[U, U]$ is a nonzero square closed $*$-Lie ideal of $R$ and $* d=d *$, we find that

$$
[v, F(v)] U d^{2}(t)=[v, F(v)] U *\left(d^{2}(t)\right)=0 \text {, for all } v \in U, t \in[U, U] \cap S_{a_{*}}(R) \text {. }
$$

By Lemma 1, we get either [v, $F(v)]=0$, for all $v \in U$, or $d^{2}(t)=0$ for each $t \in$ $[U, U] \cap S_{a_{*}}(R)$. Let $t \in[U, U]$, as $t+t^{*}, t-t^{*} \in[U, U] \cap S_{a_{*}}(R)$ and $d^{2}(t)=0$. Hence we get $d^{2}(t)=0$, for all $t \in[U, U]$. Thus we obtain that

$$
[v, F(v)]=0 \text { or } d^{2}(t)=0 \text {, for all } v \in U, t \in[U, U] .
$$

In the former case, $U \subseteq Z$ by Theorem 2. In the latter case, $[U, U] \subseteq Z$ by Lemma 5, and so $U \subseteq Z$ by Lemma 3. This completes the proof.

Proceeding on the same lines with some necessary variations in the proof of Theorem 3, we can prove the following theorem:

Theorem 4. Let $R$ be a 2-torsion free $*$-prime ring and $U$ a nonzero square closed $*-$ Lie ideal of $R$. If $R$ admits nonzero derivations $d$ which commutes with $*$ and $g$ such that $[d(u), g(v)]=0$, for all $u, v \in U$, then $U \subseteq Z$.

Theorem 5. Let $R$ be a 2 -torsion free $*$-prime ring and $U$ a nonzero square closed $*-$ Lie ideal of $R$. If $R$ admits a nonzero generalized derivation $F$ associated with nonzero derivation $d$ which commutes with $*$ such that $d(u) o F(v)=0$, for all $u, v \in U$, then $U \subseteq Z$. 
Proof. We have

$$
d(u) o F(v)=0, \text { for all } u, v \in U .
$$

Replacing $u$ by $u^{2}$ in (3.8), we get

$(d(u) o F(v)) u+d(u)[u, F(v)]+u(d(u) o F(v))-[u, F(v)] d(u)=0$, for all $u, v \in U$ and so,

$$
d(u)[u, F(v)]-[u, F(v)] d(u)=0, \text { for all } u, v \in U .
$$

We can write $[d(u),[u, F(v)]]=0$, for all $u, v \in U$ from (3.9). This yields that $\left[d(u), I_{F(v)}(u)\right]=0$, where $I_{F(v)}: R \rightarrow R, I_{F(v)}(x)=[x, F(v)]$ is an inner derivation of $R$. By Theorem 4, we get either $I_{F(v)}=0$ or $U \subseteq Z$. In the first case, $F(U) \subset Z$, and so $[u, F(u)]=0$, for all $u \in U$. This implies that $U \subseteq Z$ by Theorem 2. This completes the proof.

Theorem 6. Let $R$ be a 2-torsion free $*$-prime ring and $U$ a nonzero square closed $*$-Lie ideal of $R$. If $R$ admits nonzero derivations $d$ which commutes with * and $g$ such that $d(u) \operatorname{og}(v)=0$, for all $u, v \in U$, then $U \subseteq Z$.

Proof. Using the same arguments as in the proof of Theorem 5, we get the required result.

Theorem 7. Let $R$ be a 2 -torsion free $*$-prime ring and $U$ a nonzero square closed $*$-Lie ideal of $R$. If $R$ admits a nonzero generalized derivation $F$ associated with nonzero derivation $d$ which commutes with $*$ such that $[d(u), F(v)]=[u, v]$, for all $u, v \in U$, then $U \subseteq Z$.

Proof. Replacing $v$ by $2 v w, w \in U$ and using $\operatorname{char} R \neq 2$, we have

$$
\begin{gathered}
{[d(u), F(v)] w+F(v)[d(u), w]+v[d(u), d(w)]+[d(u), v] d(w)-[u, v] w-v[u, v]} \\
=0 .
\end{gathered}
$$

Applying hypothesis, we get

$$
F(v)[d(u), w]+v[d(u), d(w)]+[d(u), v] d(w)-v[u, w]=0, \text { for all } u, v, w \in U
$$

Writing $w$ for $d(u), u \in[U, U]$, we obtain that

$$
v\left[d(u), d^{2}(u)\right]+[d(u), v] d^{2}(u)-v[u, d(u)]=0 .
$$

Taking $2 v w$ for $v$ and using $\operatorname{char} R \neq 2$, (3.12), we see that

$$
[d(u), v] U d^{2}(u)=0 \text {, for all } v \in U, u \in[U, U] .
$$

Since $[U, U]$ is a nonzero square closed $*$-Lie ideal of $R$ and $* d=d *$, we can get $[d(u), v] U *\left(d^{2}(u)\right)=0$, for all $v \in U, u \in[U, U] \cap S_{a_{*}}(R)$. Hence we arrive at

$$
[d(u), v] U d^{2}(u)=[d(u), v] U *\left(d^{2}(u)\right)=0 \text {, for all } v \in U, u \in[U, U] \cap S_{a_{*}}(R) .
$$


By Lemma 1, we get either $[d(u), v]=0$ or $d^{2}(u)=0$, for each $u \in[U, U] \cap S_{a_{*}}(R)$. Let $u \in[U, U]$, as $u+u^{*}, u-u^{*} \in[U, U] \cap S_{a_{*}}(R)$ and $\left[d\left(u \pm u^{*}\right), v\right]=0$ or $d^{2}(u \pm$ $\left.u^{*}\right)=0$. Hence we have $[d(u), v]=0$ or $d^{2}(u)=0$, for all $u \in[U, U]$. We set

$$
K=\{u \in[U, U] \mid[d(u), v]=0, \text { for all } v \in U\}
$$

and

$$
L=\left\{u \in[U, U] \mid d^{2}(u)=0\right\} .
$$

Clearly each of $K$ and $L$ is additive subgroup of $[U, U]$. Morever, $[U, U]$ is the settheoretic union of $K$ and $L$. But a group can not be the set-theoretic union of two proper subgroups, hence $K=[U, U]$ or $L=[U, U]$. In the former case, we have $[d(u), u]=0$ for all $u \in[U, U]$ and so using Corollary $1,[U, U] \subseteq Z$. In the second case, we have $d^{2}([U, U])=0$. That is $[U, U] \subset Z$ by Lemma 5 . Hence using Lemma 3 , we get $U \subseteq Z$. This completes the proof.

Proceeding on the same lines with some necessary variations in the proof of Theorem 7, we can prove the following theorem which partially generalizes the result of [3, Theorem 1].

Theorem 8. Let $R$ be a 2-torsion free $*$-prime ring and $U$ a nonzero square closed $*$-Lie ideal of $R$. If $R$ admits nonzero derivations $d$ which commutes with * and $g$ such that $[d(u), g(v)]=[u, v]$, for all $u, v \in U$, then $U \subseteq Z$.

Theorem 9. Let $R$ be a 2-torsion free $*$-prime ring and $U$ a nonzero square closed $*$-Lie ideal of $R$. If $R$ admits a nonzero generalized derivation $F$ associated with nonzero derivation $d$ which commutes with $*$ such that $[d(u), F(v)]=-[u, v]$, for all $u, v \in U$, then $U \subseteq Z$.

Proof. It can be proved using the techniques of Theorem 7.

Theorem 10. Let $R$ be a 2-torsion free $*-$ prime ring and $U$ a nonzero square closed $*-$ Lie ideal of $R$. If $R$ admits a nonzero generalized derivation $F$ associated with nonzero derivation $d$ which commutes with $*$ such that $d(u) o F(v)=u o v$, for all $u, v \in U$, then $U \subseteq Z$.

Proof. By the hypothesis, we have

$$
d(u) o F(v)-u o v=0, \text { for all } u, v \in U .
$$

Replacing $v$ by $2 v w, w \in U$ in (3.15), we get

$$
\begin{gathered}
2((d(u) o F(v)) w-F(v)[d(u), w]+(d(u) o v) d(w)-v[d(u), d(w)] \\
-(u o v) w+v[u, w])=0 .
\end{gathered}
$$

Using (3.15) and $\operatorname{char} R \neq 2$, we obtain

$-F(v)[d(u), w]+(d(u) o v) d(w)-v[d(u), d(w)]+v[u, w]=0$, for all $u, v, w \in U$. 
Substituting $d(u), u \in[U, U]$ for $w$ in (3.16), we have

$(d(u) o v) d^{2}(u)-v\left[d(u), d^{2}(u)\right]+v[u, d(u)]=0$, for al $u \in[U, U], v, w \in U$.

Replacing $v$ by $2 w v, w \in U$ in (3.17), we get

$2\left(w(d(u) o v) d^{2}(u)+[d(u), w] v d^{2}(u)-w v\left[d(u), d^{2}(u)\right]+w v[u, d(u)]=0\right.$.

In the view of (3.17), the above expression yields that $2[d(u), w] v d^{2}(u)=0$, for all $u \in[U, U], v, w \in U$. Since $\operatorname{char} R \neq 2$, we arrive at

$$
[d(u), w] U d^{2}(u)=0 \text {, for all } u \in[U, U], w \in U .
$$

The proof is comleted by using the same arguments in the equation (3.13) of Theorem 7 .

Corollary 2. Let $R$ be a 2-torsion free $*$-prime ring and $U$ a nonzero square closed $*-$ Lie ideal of $R$. If $R$ admits nonzero derivations $d$ which commutes with $*$ and $g$ such that $d(u) \operatorname{og}(v)=u o v$, for all $u, v \in U$, then $U \subseteq Z$.

Proof. Using the same arguments as in the proof of Theorem 10, we get

$$
[d(u), w] U g d(u)=0 \text {, for all } u \in[U, U], w \in U .
$$

Using $* d=d *$, we arrive at

$$
*([d(u), w]) U g d(u)=0, \text { for all } u \in[U, U], w \in U .
$$

Arguing in the similar manner as we have done in the proof of Theorem 10, we arrive at $[d(u), u]=0$ or $g d(u)=0$, for all $u \in[U, U]$. In the former case, we have $U \subseteq Z$ by Corollary 1 . In the second case, using Theorem 1, we find $U \subseteq Z$.

Theorem 11. Let $R$ be a 2-torsion free $*$-prime ring and $U$ a nonzero square closed $*-$ Lie ideal of $R$. If $R$ admits a nonzero generalized derivation $F$ associated with nonzero derivation $d$ which commutes with $*$ such that $d(u) o F(v)=-u o v$, for all $u, v \in U$, then $U \subseteq Z$.

Proof. Similarly Theorem 10.

Theorem 12. Let $R$ be a 2-torsion free $*$-prime ring and $U$ a nonzero square closed $*-$ Lie ideal of $R$. If $R$ admits a nonzero generalized derivation $F$ associated with nonzero derivation $d$ which commutes with $*$ such that $d(u) F(v)-u v \in Z$, for all $u, v \in U$, then $U \subseteq Z$.

Proof. By the hypothesis, we have

$$
d(u) F(v)-u v \in Z \text {, for all } u, v \in U .
$$

Replacing $v$ by $2 v w, w \in U$ in (3.18), we get

$$
2((d(u) F(v)-u v) w+d(u) v d(w)) \in Z \text {, for all } u, v, w \in U .
$$


Since $\operatorname{char} R \neq 2$, we have

$$
(d(u) F(v)-u v) w+d(u) v d(w) \in Z, \text { for all } u, v, w \in U .
$$

Commutting this term with $w$ and using the hypothesis, we obtain

$$
[d(u) v d(w), w]=0, \text { for all } u, v, w \in U .
$$

Substituting $2 d(u) v, u \in[U, U]$ for $v$ in (3.20) and using this equation, we get

$$
[d(u), w] d(u) U d(w)=0 \text {, for all } w \in U, u \in[U, U] .
$$

Since $U$ is a nonzero square closed $*$-Lie ideal of $R$ and using $* d=d *$ in the last equation, we arrive at

$$
\begin{gathered}
{[d(u), w] d(u) U d(w)=[d(u), w] d(u) U *(d(w))=0,} \\
\text { for all } u \in[U, U], w \in U \cap S_{a_{*}}(R) .
\end{gathered}
$$

Arguing in the similar manner as we have done in the proof of Theorem 10 after (3.18) yields that either $[d(u), w] d(u)=0$ or $d(w)=0$, for each $w \in U, u \in[U, U]$.

Now, we assume that $[d(u), w] d(u)=0$, for all $w \in U, u \in[U, U]$. Replacing $w$ by $2 w v, v \in U$ in this equation and using this, we have

$$
[d(u), w] v d(u)=0, \text { for all } u \in[U, U], w, v \in U .
$$

Again using $[U, U]$ is a nonzero square closed $*$-Lie ideal of $R$ and $* d=d *$ in the last equation, we get

$$
[d(u), w] v d(u)=[d(u), w] v *(d(u))=0 \text {, for all } u \in U, w, v \in[U, U] .
$$

An application of similar techniques as used in the proof of Theorem 10, we obtain that $U \subseteq Z$.

Corollary 3. Let $R$ be a 2-torsion free $*$-prime ring and $U$ a nonzero square closed $*-$ Lie ideal of $R$. If $R$ admits nonzero derivation $d$ which commutes with * such that $d(u) d(v)-u v \in Z$, for all $u, v \in U$, then $U \subseteq Z$.

Theorem 13. Let $R$ be a 2 -torsion free $*-$ prime ring and $U$ a nonzero square closed $*$-Lie ideal of $R$. If $R$ admits a nonzero generalized derivation $F$ associated with nonzero derivation $d$ which commutes with $*$ such that $d(u) F(v)+u v \in Z$, for all $u, v \in U$, then $U \subseteq Z$.

Proof. Similarly.

Corollary 4. Let $R$ be a 2-torsion free $*$-prime ring and $U$ a nonzero square closed $*-$ Lie ideal of $R$. If $R$ admits nonzero derivation $d$ which commutes with $*$ such that $d(u) d(v)+u v \in Z$, for all $u, v \in U$, then $U \subseteq Z$.

The following example demonstrates that the above results are not true in the case of arbitrary rings.

Example 14. Let $I$ be the ring of integers. Set $R=\left\{\left(\begin{array}{cc}a & b \\ 0 & c\end{array}\right) \mid a, b, c \in I\right\}$ 
and $U=\left\{\left(\begin{array}{ll}0 & b \\ 0 & 0\end{array}\right) \mid b \in I\right\}$. We define the following maps: $*\left(\begin{array}{cc}a & b \\ 0 & c\end{array}\right)=$ $\left(\begin{array}{cc}c & -b \\ 0 & a\end{array}\right), F\left(\begin{array}{cc}a & b \\ 0 & c\end{array}\right)=\left(\begin{array}{cc}a & 2 b \\ 0 & 0\end{array}\right), d\left(\begin{array}{cc}a & b \\ 0 & c\end{array}\right)=\left(\begin{array}{ll}0 & b \\ 0 & 0\end{array}\right)$. Then it is easy to see that $U$ is a nonzero square closed $*$-Lie ideal of $R$ and $F$ is a generalized derivation associated with a nonzero derivation $d$ commuting with $*$. Moreover, it is straightforward to check that $F$ satisfies the properties: 1) $[F(u), u]=0$, 2) $[d(u), F(v)]=0,3) d(u) o F(v)=0,4)[d(u), F(v)]= \pm[u, v], 5) d(u) o F(v)=$ $\pm u o v, 6) d(u) F(v) \pm u v \in Z$, for all $u, v \in U$. However, $U \nsubseteq Z(R)$.

\section{REFERENCES}

[1] M. Ashraf, A. Ali, and R. Rani, "On generalized derivations of prime rings," Southeast Asian Bull. Math., vol. 29, no. 4, pp. 669-675, 2005.

[2] M. Ashraf and A. Khan, "Commutativity of $*$-prime rings with generalized derivations," Rend. Semin. Mat. Univ. Padova, vol. 125, pp. 71-79, 2011.

[3] H. E. Bell and M. N. Daif, "On commutativity and strong commutativity-preserving maps," Can. Math. Bull., vol. 37, no. 4, pp. 443-447, 1994.

[4] M. Brešar, "On the distance of the composition of two derivations to the generalized derivations," Glasg. Math. J., vol. 33, no. 1, pp. 89-93, 1991.

[5] L. Oukhtite and S. Salhi, "On commutativity of $\sigma$-prime rings," Glas. Mat., III. Ser., vol. 41, no. 1, pp. 57-64, 2006.

[6] L. Oukhtite and S. Salhi, "Lie ideals and derivations of $\sigma$-prime rings," Int. J. Algebra, vol. 1, no. 1-4, pp. 25-30, 2007.

[7] L. Oukhtite and S. Salhi, "On generalized derivations of $\sigma$-prime ring," Afr. Diaspora J. Math., vol. 5, no. 1, pp. 21-25, 2007.

[8] L. Oukhtite and S. Salhi, "Centralizing automorphisms and Jordan left derivations on $\sigma$-prime rings," Adv. Algebra, vol. 1, no. 1, pp. 19-26, 2008.

[9] L. Oukhtite, S. Salhi, and L. Taoufiq, "Commutativity conditions on derivations and Lie ideals in $\sigma$-prime rings," Beitr. Algebra Geom., vol. 51, no. 1, pp. 275-282, 2010.

[10] E. C. Posner, "Derivations in prime rings." Proc. Am. Math. Soc., vol. 8, pp. 1093-1100, 1958.

\section{Authors' addresses}

\section{Shuliang Huang}

Chuzhou University, Department of Mathematics, Chuzhou Anhui, 239012, P. R. China,

E-mail address: shulianghuangesina.com

\section{Öznur Gölbaşı}

Cumhuriyet University, Faculty of Science, Department of Mathematics, 58140, Sivas - TURKEY

E-mail address: ogolbasi@cumhuriyet.edu.tr 